\title{
ENHANCING THE EFFICIENCY OF INDOOR LOCAL VENTILATION USING CROSS-FLOW FANS: CASE STUDY FOR A SMOKING CABIN
}

\author{
ANDREI DRAGOMIRESCU ${ }^{1}$, ADRIAN CIOCĂNEA ${ }^{1 *}$ \\ 1 "Politehnica" University of Bucharest, Department of Hydraulics, Hydraulic Machinery \\ and Environmental Engineering, 313 Splaiul Independentei, Bucharest, 060042, Romania
}

\begin{abstract}
The paper presents an original solution for increasing air quality and reducing energy consumption of the local indoor ventilation by using cross-flow fans. The solution is a combination between the local exhaust ventilation (LEV) technique from industry, negative-pressure isolation rooms used in hospitals, and air curtains (AC) used for isolating of indoor/outdoor spaces. The solution provides a high air flow rate at low velocity due to the high value of the flow coefficient of the cross flow fans and, in the same time, allows modular setup according to local space geometry. A case study is proposed regarding the isolation of a smoking area where smell and airborne particles appear. A 3D numerical simulation was performed, in which one cross-flow fan with long axial length was considered. The optimum air flow rate and flow pattern was obtained in order to isolate the local space. The results show that a new approach for reducing sick building syndrome could be addressed by providing modular and local ventilation using cross-flow fans.
\end{abstract}

Keywords: air quality, indoor ventilation, cross-flow fans, air curtains, sick building syndrome

\section{INTRODUCTION}

In the last years, the requirements imposed to ventilation systems became more and more complex. The increase in interior comfort in buildings destined mainly for public activities - by heating, cooling, conditioning - as well as the demand for improving their energy efficiency - by thermal insulation, heat recovery - required new studies regarding the quality of indoor air. An inadequate design or operation of a ventilation system can cause a dramatic reduction in indoor air quality (IAQ). The terms used to describe the effect of the reduction in IAQ are sick building syndrome (SBS), tight building syndrome, and building related illness [1-3]. An analysis of the solutions to the aforementioned ventilation problems indicates that, regardless of the emissions considered, specific to closed buildings with or without underground parking, the systems used are based on:

1) supplying fresh air through vents placed according to indoor architecture [4, 5];

2) directing air currents by using air curtains formed usually in entry or exit sections, with the purpose of isolating indoor spaces [6-9];

3) partitioning indoor spaces by introducing screens for directing the air flow between entry and exit sections $[4,10]$.

Regarding the results related to the most efficient methods for local ventilation, there are different studies concerning the protection of workers from different industrial fields [11-13], the insulation of hazardous

\footnotetext{
* Corresponding author, email: adrian.ciocanea@upb.ro

(C) 2015 Alma Mater Publishing House
} 
spaces - mainly in hospitals - [14-17], or the reduction of the effects of sick building syndrome both in public [18] and in private buildings [19].

In this paper all three aspects of local ventilation are considered: extraction of polluted air close to source, creation of an optimal negative gauge pressure in order to isolate the pollution source from the rest of the space, and placing the vents in the most appropriate locations in order to evacuate the most part of the agents that cause the sick building syndrome (VOC, molds, ozone from some office machines, etc.). For the purpose of validating the hypothesis according to which local ventilation in public buildings can be of modular type and customized in order to counteract to the largest extent the sick building syndrome, a numerical simulation of a smoking cabin is proposed. This example was chosen because it allows verifying the evacuation of both smoke and airborne particles, which is close to the real case of an office building. For the ventilation system of the smoking cabin a cross-flow fan was chosen due to the fact that such a fan has the advantage of low power consumption at the high air flow rates and velocities within the standardized limits for indoor ventilation.

In the following, the problem is formulated in terms of geometry, parameters, equations and boundary conditions, and numerical algorithms. Results are then presented and discussed and conclusions are drawn.

\section{PROBLEM FORMULATION}

The smoking cabin studied by numerical simulations is presented in Figure 1. Its main dimensions - width, depth, and height - are given in $\mathrm{mm}$. The cabin consists of a semi-open smoking room and a closed filter room. The smoking room is enclosed by two lateral walls, a back wall, and a ceiling and is opened to the room where the smoking cabin is placed. The ceiling contains in the middle a compartment with two lateral suction slots through which air and cigarette smoke are absorbed by the filtering system through a suction duct placed above the ceiling. The length of the suction slots roughly equals the depth of the smoking room, their height is of $50 \mathrm{~mm}$. The floor of the smoking room is raised to accommodate a cross-flow fan that blows a plane free air jet upwards at a small flow rate through an exhaust slot placed at the edge of the floor. The exhaust slot extends along the entire opening of the smoking room and has a width of $50 \mathrm{~mm}$. A small table is installed inside the smoking room for supporting the ashtray and a few glasses or coffee cups. The filter room is a cabinet that contains a suction fan for absorbing air and cigarette smoke at the ceiling of the smoking room, filters for retaining the smoke and airborne particles, and additional equipment that might be required.

The smoking cabin is placed by a wall of a larger room - the back vertical surface in Figure 1. This room, which is only partly contained inside the computational domain, will be denoted in the following as main room. The part of the main room that contains the smoking cabin has a height of $3.5 \mathrm{~m}$, a width of $6 \mathrm{~m}$ (measured along the back wall by which the cabin is placed), and a depth of $4 \mathrm{~m}$ (measured from front to back in Figure 1). In a first approximation, the presence of humans inside the smoking cabin was neglected.

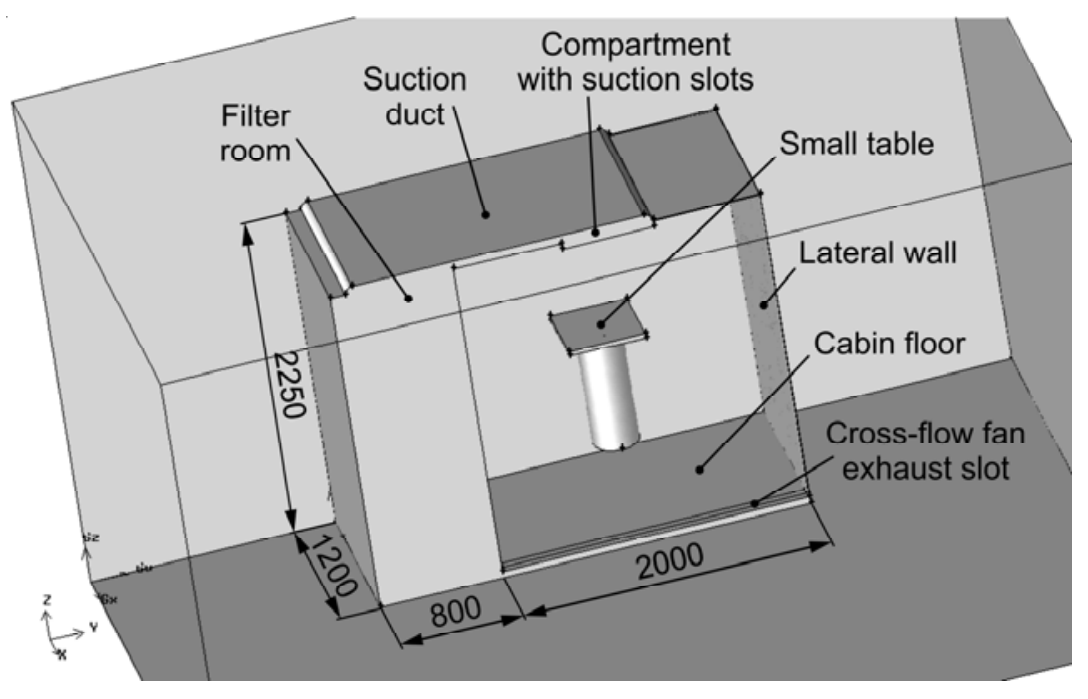

Fig. 1. Sketch of the smoking cabin. 


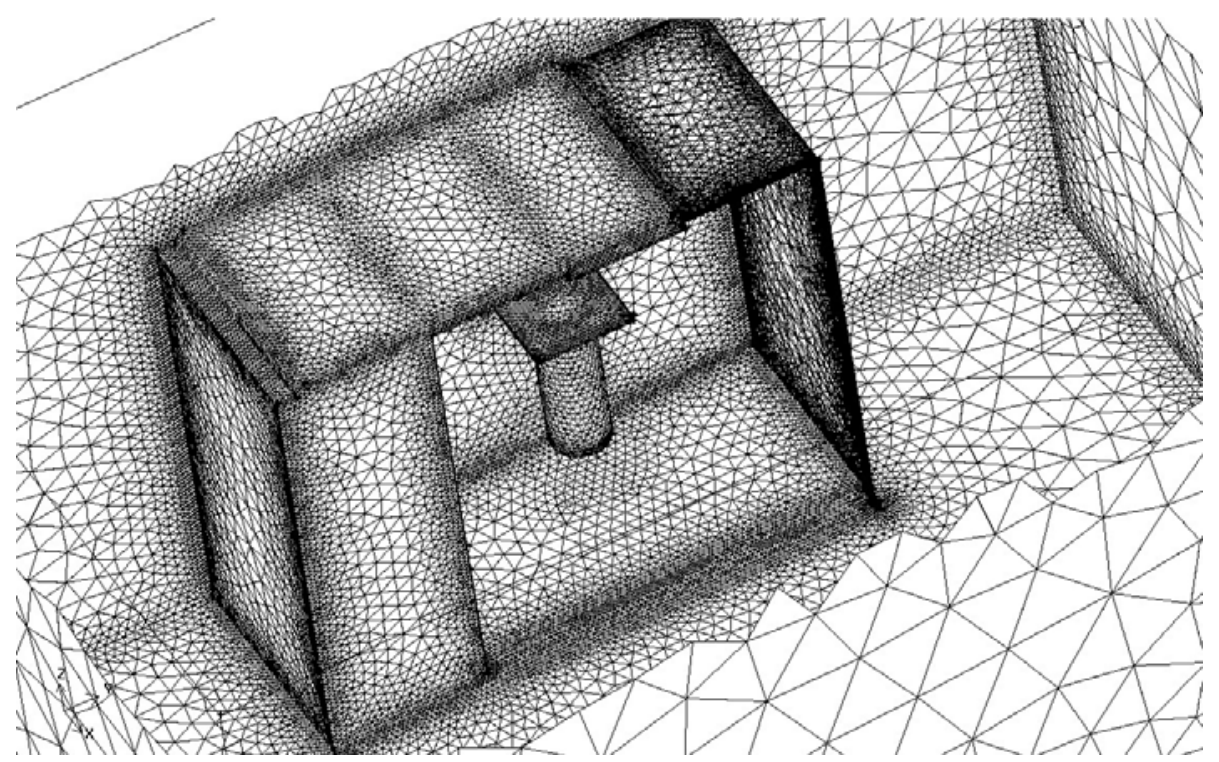

Fig. 2. Mesh on the boundaries of the computational domain.

The computational domain was meshed with a total number of 725313 tetrahedral cells in the middle and prismatic cells of boundary layer type at the walls. Figure 2 presents the mesh on the boundaries of the computational domain.

The air was considered a Newtonian incompressible fluid and its flow was considered to be in steady state. Possible heat sources were not taken into consideration, therefore the problem was treated as isothermal. As absolute reference pressure and reference temperature the values $p_{0}=10^{5} \mathrm{~Pa}$ and $t_{0}=25^{\circ} \mathrm{C}$, respectively, were used. At these conditions, air has the density $\rho=1.1678 \mathrm{~kg} / \mathrm{m}^{3}$ and the dynamic viscosity $\mu=1.8465 \times 10^{5} \mathrm{~Pa}$.s.

The steady, incompressible, turbulent flow is described by the continuity equation, the momentum equations and the equations of the turbulence model. To describe turbulence, the SST $k-\omega$ model was chosen due to the fact that it is adequate for studying flow that involve plane free jets [20] like that created by the cross-flow fan. Moreover, the SST $k-\omega$ model seems to be appropriate in general for modeling indoor air flow [21].

The usual condition of no-slip was used at the solid walls. At the exhaust slot of the cross-flow fan a mass flow rate of $0.035 \mathrm{~kg} / \mathrm{s}$ was imposed. The evacuation of the same mass flow rate was set as boundary condition at the two suction slots. The value of the mass flow rate was chosen so that the average exhaust velocity - or initial jet velocity - of about $0.3 \mathrm{~m} / \mathrm{s}$ remains within the limits accepted for mechanical ventilation. On the surface that separates the computational domain from the rest of the main room (the vertical front surface in Figure 1) a gauge pressure of 0 Pa was set.

The governing equations together with the boundary conditions were integrated numerically using the pressure based segregated solver implemented in Fluent. The SIMPLE scheme was chosen for the pressure-velocity coupling. The momentum equations were discretized in space with the second order upwind scheme. The first order upwind scheme was used for the equations of the turbulence kinetic energy, $k$, and of its specific dissipation rate, $\omega$. It was considered that convergence was attained when the residuals of all equations dropped below $10^{-3}$.

\section{RESULTS AND DISCUSSION}

Of main interest it was to verify whether smoke and airborne particles can escape from the smoking cabin to the main room. For this purpose, based on the results of the numerical simulations, pathlines of particles injected at different locations inside the smoking cabin were calculated and plotted for analysis.

Figure 3 shows pathlines obtained for particles injected at equally spaced positions along the exhaust slot of the cross-flow fan. For a better visibility, two views of the same pathlines are presented. One view is from the left of the cabin and above the ceiling, the other view is from the left and below the floor. The shapes of the pathlines 


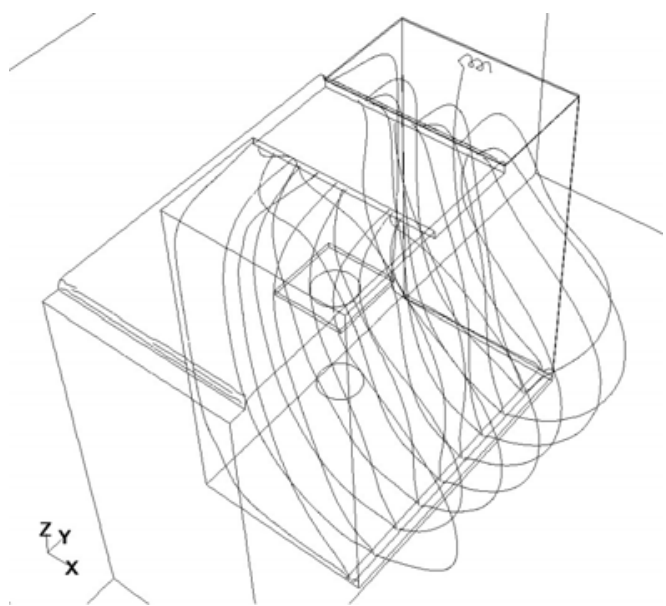

a)

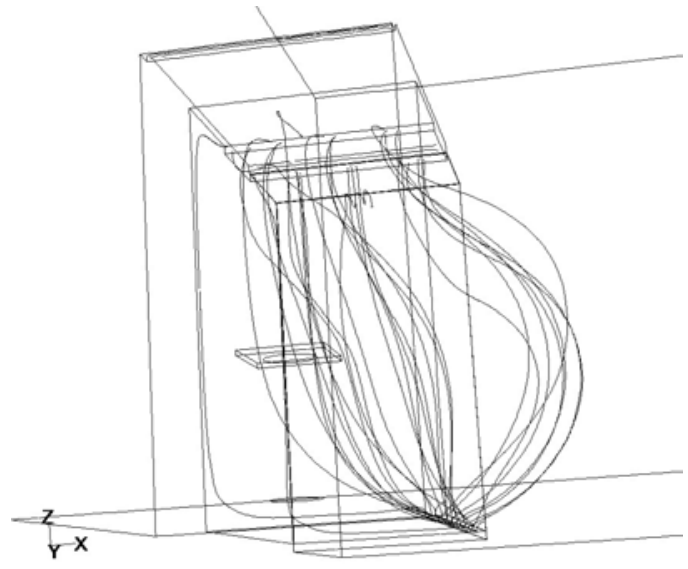

b)

Fig. 3. Pathlines of particles injected along the exhaust slot of the cross-flow fan: a) view from the left and above, b) view from the left and below.

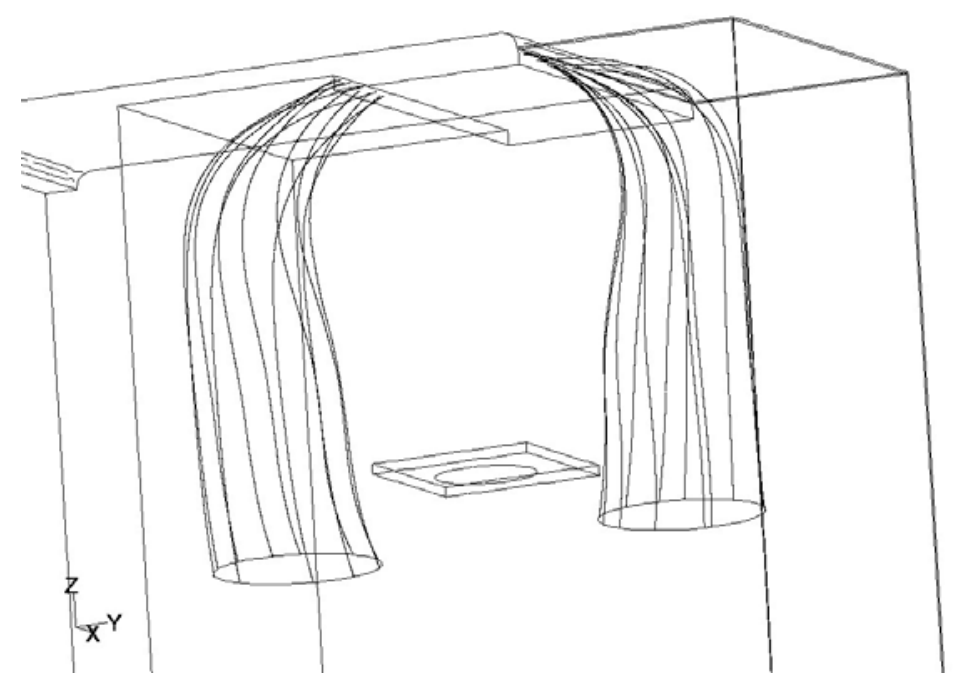

Fig. 4. Pathlines of particles injected along two circles at $0.8 \mathrm{~m}$ above cabin floor.

show that the plane free jet created by the cross-flow fan spreads rapidly. This could be easily explained considering the very low initial velocity of the jet. However, the bent pathlines suggest that the negative gauge pressure created at the suction slots in the cabin ceiling is large enough to create the driving forces that direct all particles towards these suction slots, so that no particle escapes to the main room.

The behavior of cigarette smoke inside the cabin was investigated considering that smoke is produced by two smokers. For a first case, the less probable situation was studied, when the two smokers would keep their cigarettes down, relatively close to their bodies. For this purpose, particles were injected along two circles having a diameter of $0.5 \mathrm{~m}$ and lying on a horizontal plane at $0.8 \mathrm{~m}$ above the cabin floor to the left and right of the table. The pathlines obtained are presented in Figure 4. As it can be seen, all particles move towards the suction slots, having no tendency to escape outside of the smoking cabin.

The case was then considered when the smokers would rest their hands on the table, keeping the cigarettes close above it. For this case, particles were injected along a circle that has a diameter of $0.5 \mathrm{~m}$ and is placed at $0.2 \mathrm{~m}$ above the table. The resulting pathlines are presented in Figure 5. It is clear that all particles are absorbed by the suction slots. It is interesting to note that the three particles injected close to the back wall of the cabin have a more complicated path. They first descend towards the table and then move almost horizontally towards the entrance of the cabin. This behavior suggests that the complex flow inside the cabin generates descendant air 


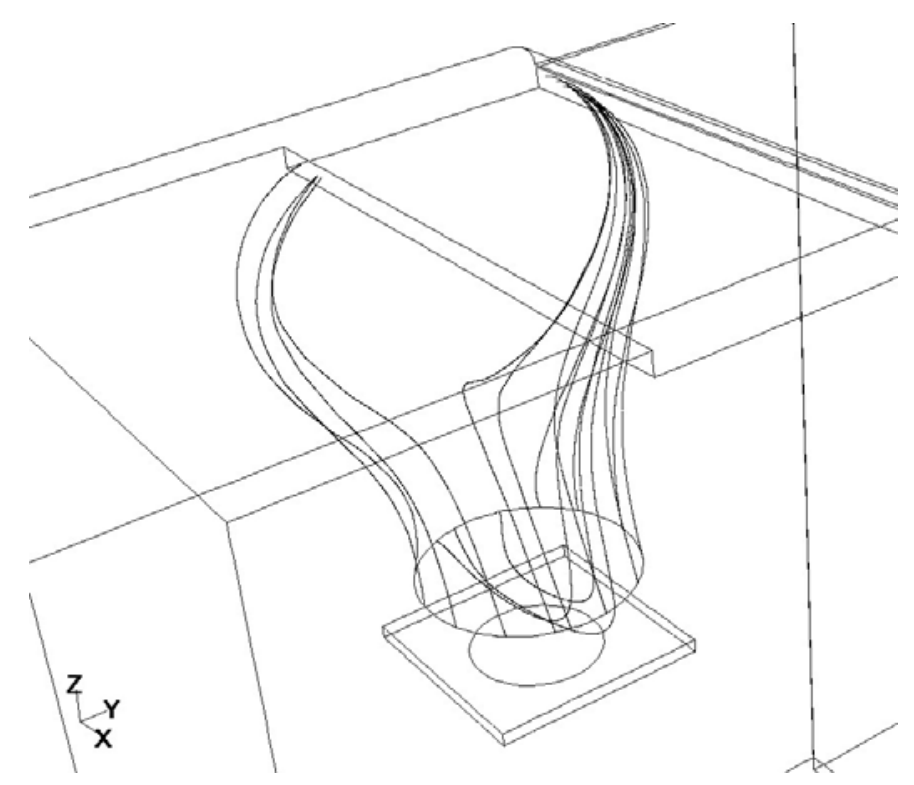

Fig. 5. Pathlines of particles injected along a circle at $0.2 \mathrm{~m}$ above the table.

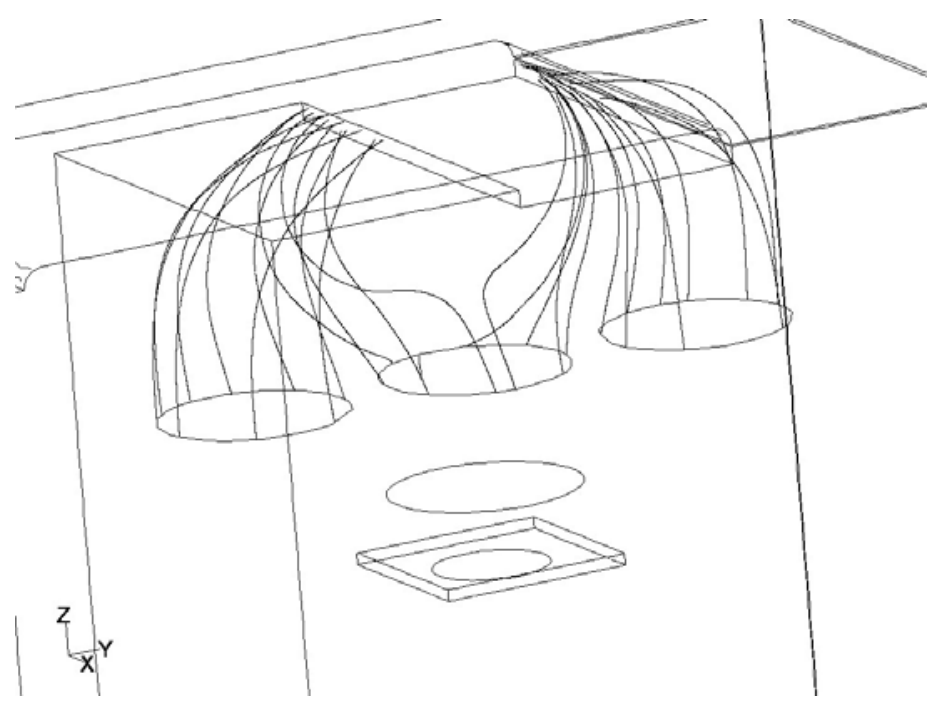

Fig. 6. Pathlines of particles injected along two circles at $1.5 \mathrm{~m}$ above the floor, to the left and right of the table, and along a third circle at $0.5 \mathrm{~m}$ above the table.

currents close to the back wall. However, the particles do not escape from the cabin, being trapped and directed towards the suction slots in the ceiling by upward air currents generated by the combination between the plane free jet coming from below and the negative gauge pressure at the suction slots.

A final case was considered when the smokers would keep the cigarettes at mouth level above the table and, at the same time, would slowly exhale smoke. The exhaling velocity was neglected in this study. Particles were injected along three circles of $0.5 \mathrm{~m}$ in diameter. Two of the circles are situated at $1.5 \mathrm{~m}$ above the cabin floor and roughly approximate the perimeter where smoke could be exhaled. The third circle is situated at $0.5 \mathrm{~m}$ above the table where the cigarettes would be briefly kept.

Figure 6 presents the pathlines obtained. It can be seen that all particles are absorbed by the suction slots. Considering the results presented previously, this behavior is to be expected since the injection points are quite close to the suction slots. 


\section{CONCLUSIONS}

In this paper, the original solution of using a smoking cabin for increasing air quality and reducing the energy consumption of indoor ventilation was presented. The originality of the solution comes from the fact that the cabin uses a cross-flow fan to create a plane free jet that acts as an air curtain for isolating the inside of the cabin from the main room. The complex flow inside the smoking cabin and in its immediate vicinity, generated by the combination between the plane free jet and the negative gauge pressure created at suction slots on cabin ceiling, was investigated by means of numerical simulations. After obtaining a converged solution for the flow, particles were injected at the exhaust slot of the cross-flow fan and at different positions inside the cabin and their pathlines were calculated in order to verify whether such particles can escape from the cabin. The results presented suggest that smoke and airborne particles cannot escape outside of the cabin, which allows us to conclude that the solution proposed is highly efficient.

\section{REFERENCES}

[1] WHO, Indoor Air quality research, EURO-reports and studies 103, WHO-Regional Office for Europe, Copenhagen, 1984.

[2] Sundell, J., What we know, and don't know about sick building syndrome, ASHRAE Journal 1996, p. 51-7.

[3] Gupta, S., Khare, M., Goyal, R., Sick building syndrome-A case study in a multistorey centrally airconditioned building in the Delhi City, Building and Environment, vol. 42, 2007, p. 2797-2809.

[4] Leea, H., Awbi, H.B., Effect of internal partitioning on indoor air quality of rooms with mixing ventilationbasic study, Building and Environment, vol. 39, 2004, p. 127-141.

[5] Nielsen, J.R., Nielsen, P.V., Svidt, K., Air distribution in a furnished room ventilated by mixing ventilation, Proceedings of the Ventilation '97: Global Developments in Industrial Ventilation, Ottawa, Canada, 1997.

[6] Foster, A.M., Swaina, M.J., Barrettb, R., Jamesa, S.J., Experimental verification of analytical and CFD predictions of infiltration through cold store entrances, International Journal of Refrigeration, vol. 26, 2003, p. 918-925.

[7] Foster, A.M., Swain, M.J., Barrett, R., D’Agaro, P., Ketteringham, L.P., James, S.J., A Three-dimensional effects of an air curtain used to restrict cold room infiltration, Applied Mathematical Modelling, vol. 31, 2007, p. $1109-1123$.

[8] Gugliermetti, F., Santarpia, L., Zori, G., Air curtain applied to fire smoke pollution control, AIR pollution 2003, 11th international conference on modelling, monitoring and management of air pollution, Catania, Italy, 2003, p. 541-549.

[9] Pavageau, M., Nieto, E., Rey, C., Odour and VOC confining in large enclosures using air curtains, Water Science and Technology, vol. 44, no. 9, 2001, p. 165-171.

[10] Sakurai, H., Hayashi, T., Shibata, M., Kanehara, K., Researches on air shutter for fire defence, Fire Safety Journal, vol. 80, no. 2, 1979, p. 9-16.

[11] Caputo, A., Pelagagge, P., Upgrading mixed ventilation systems in industrial conditioning, Applied Thermal Engineering, vol. 29, 2009, p. 3204-3211.

[12] Hasan, B.H., Said, M.R., Leman, A.M., Mokhtar, A.M., The Performance Test on Local Exhaust Ventilation (LEV) System to Prevent Chlorine Gas Leakage in Water Treatment Plant, International Journal of Environmental Science and Development, vol. 4, no. 4, 2013.

[13] Norerama, B., Pagukuman, D., Mutalib, A.L., Mohammad, Z.Y.M., The Efficacy of Local Exhaust Ventilation (LEV) System Controls on Aerosols Exposures during Aluminum Cans Production, Applied Mechanics and Materias, vol. 465-466, 2013, p. 438-442.

[14] Fletcher, A., Booth, W., Arribas, B.B., Validation of a Neutral Pressure Isolation Room, Proceedings of Clima 2007 WellBeing Indoors, The 9th Congress, Helsinki, Finland, 2007.

[15] Booth, W., Arribas, B.B., Noakes, C., Fletcher, L., Sleigh, A., Tomlinson, N., Characterizations of the protection provided by the ventilation strategy in hospital isolation rooms, Healthy Buildings Conference, Syracuse, USA, 2009.

[16] Carla, B., Pietro, L., Modelling infection spreading control in a hospital isolation Room, Journal of Biomedical Science and Engineering, vol. 3, 2010, 653-663.

[17] Fisk, W. J., Mirer, A.G., Mendell, M.J., Quantitative relationship of sick building syndrome symptoms with ventilation rates, Indoor Air, vol. 19, no. 2, 2009, p. 159-165. 
[18] Falidah, N., Juliana, J., Indoor Air Quality (IAQ) and Sick Buildings Syndrome (SBS) among Office Workers in New and Old Buildings in University Putra Malaysia, Serdang, Health and Environment Journal, vol. 3, no. 2, 2012, p. 98-109.

[19] Dimitroulopoulou, C., Ventilation in European dwellings: A review, Building and Environment, vol. 47, 2012, p. 109-125.

[20] Fluent Inc., Fluent 6.3 User's Guide, Fluent Inc., Lebanon, 2003.

[21] Stamou, A., Katsiris, I., Verification of a CFD model for indoor airflow and heat transfer, Building and Environment, vol. 41, 2006, p. 1171-1181. 